Hoff, as modified by Morse and Frazer, but at high concentrations the divergence between the two equations is very great.

An exact form is obtained for the mass law in concentrated perfect solutions.

Boston, March 2, 1905 .

[CONTRIBUTION FROM ROGER's LABORATORY OF PHYSTCS OF THE MASSACHUSETTS INSTITLTE OH TECHNOR.OOY]

\title{
THE INDESTRUCTIBILITY OF MATTER AND THE ABSENCE OF EXACT RELATIONS AMONG THE ATOMIC WEIGHTS.
}

H: DANELT. F. CONSTOCK.

keceived March 9 rops

The two chici reasons briefly stated for believing in the evolution of the elements one from another are, first, that some such process is un loubtedly taking place in the case of the radioactive substances, while we are being forced toward the conclusion that all the elements are radio active to some degree; and second, that in the hottest stars only two known elements occur, namely, hydroget and helium, while as we pass successively to cooler and cooler stars the other elements gradually make their appearance in a more or less orderly manner. Apparently this can only mean that at these transcendental temperatures the forces due to molecular or atomic impact are comparable with the interatomic forces involved in the breaking up of one element to form another, and hence the combination necessary for the formation of the heavier ele. ments can take place only after the temperature has sufficiently dropped.

There is one seemingly fatal objection, howerer, to any rery simple statement of the evolutionary theory and this objection has not been sufficiently emphasized. The diffeulty is this, that so far as we know there are no exact simple relations between the various atomic weights, whereas if we are to assume, as the simplest form of the evolutionary theory does, that the lighter elements come from an atomic disintegration of the heavier ones, or vice versa, it is evident that simple additive relations must exist.

As we know, many simple, additive relations do exist, but they are approximate, not exact, and the deviations from exactness, though sniall, are larger than we can explain from error in atomic weight determinations.

On the basis of common conceptions, therefore, the eridence seems contradictory, certain facts seeming to require the simple evolutionary idea, while another fact, the inexactness refered to above seming 6 deny it.

I wish to show that on the basis of the clectrical constitution at mat ter this inexactness is not only to be explained but it is to be expected 
By the electrical constitution of matter is meant merely that conception, which has grown in favor among physicists of late, which considers the atom to consist wholly or in part of a group of electric charges. Some of these make themselves evident in an ionized electrolyte or in a gas, but in general the fact that the two electricities, positive and negative, are present in equal amounts makes the atom neutral as regards action on an outside point.

Now since moving charges always act like currents, and hence must set up magnetic fields, it must follow that when an apparently uncharged atom is set in motion there will be set up inside of it a magnetic field, which depends for its strength and distribution on the number, position, and magnitude of whatever charges the atom may be supposed to contain. It will therefore require more energy to set the atom in motion because of the necessity of building up this field, and hence the atom will have an added inertia, $i$. e., an added mass because of these charges.

Now it has been shown mathematically by the author (Phil. Mag., Jan., 1908), without making any assumption as regards the structure of the atom, that this added mass due to the electric charges is strictly proportional to the total electrical energy contained in the atom.

By electrical energy is meant the energy which we know must exist in space wherever there are electric lines of force. Two charges, one positive and one negative, attract each other, and if these are separated to a distance, work must be done against their mutual attraction. Since the charges themselves have changed in no way, the energy put in must exist in the surrounding space in a form generally known as "strain in the ether." Magnetic lines of force also correspond to energy stored in space and the statement proved in the paper mentioned above is that the added mass which an atom possesses because of the electric charges which it is supposed to contain depends only on the total electric and magnetic energy which the atom contains and is directly proportional to this energy.

The expression found may be written

$$
M=\frac{4}{3} \frac{E}{V^{2}}
$$

where $\mathrm{M}$ is this "electric mass," $\mathrm{V}$ is the velocity of light, and $\mathrm{E}$ is the total contained electromagnetic energy of the kind described above.

The amount of energy which corresponds to a gram mass is enormous. It is readily calculated from the formula, and is found to be $7 \times 10^{20}$ ergs, approximately, so we may make the statement that, on the present bases, the inertia of a gram mass is due to the existence within it of $7 \times 10^{20}$ ergs of confined energy.

Now, when the atom of an element breaks up the process is a violent one and a large quantity of energy is lost, i. e., goes ultimately into heat. 
Hence, if this is electric energy lost -and on the basis of the electrical conception it is electrical energy-it follows from the above that there must have been a loss in mass accompanying the atomic disintegration be. cause of the energy lost. Hence, taking the simple case of an atom of $A$, splitting up violently and forming atoms of the elements $B$ and $C$. we would find that the mass of $B$ and $C$, taken together, would be a lit the less than the mass of $A$, from which they came, the difference corre sponding to the loss of mass accompanying the loss in energy.

Now the loss of energy when an atom of radium breaks up is known. and calculation shows that this would give a loss in mass which would be of about the same size as the deviations in atomic mass or weight $\rightarrow$ common in the table.

In an important paper ${ }^{-1}$ Rydberg has shown that the atomic weights of the first twenty-seven elements of the periodic system approximate $w$ whole numbers very much more closely than chance could bring about He has also shown that the atomic weights of these elements are best considered as the sum of two parts $(N+D)$, where $N$ is an integer and $D$ is a fraction, in general positive and smaller than unity. If $M$ is the number of the element in the system (called by Rydberg the "Ordnungszahl"), then $\mathrm{N}$ is equal to $2 \mathrm{M}$ for the elements of even valence and $2 \mathrm{MI}$. 1 for the elements of odd valence. Below is given a table showing the various quantities. I have used, however, the International Atomic Weight values for 1907 instead of those Rydberg used

\begin{tabular}{|c|c|c|c|c|c|c|c|c|c|c|c|}
\hline & & & $\mathrm{N}$ & & & & & & $\therefore$ & & \\
\hline Sign. & $M$ & $2 \mathrm{M}$ & $\widehat{2 M I}$ & $\begin{array}{l}\text { A tomic } \\
\text { weight }\end{array}$ & I. & sign. & $M$. & $2 . \mathrm{X}$ & $2 \mathrm{M} \therefore 1$ & $\begin{array}{l}\text { Atomic } \\
\text { weight }\end{array}$ & I'. \\
\hline He. & 2 & + & & + & & $p$ & 1.5 & & il & $3 x$ & : \\
\hline$\ldots$ & 3 & & $\because$ & -03 & 003 & 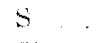 & 16 & $\therefore$ & & 32.00 & 3.60 \\
\hline$\ldots$ & + & $s$ & & 9.1 & 1.1 & C1. & $1:$ & & 35 & $.35-45$ & $6+5$ \\
\hline B. & 5 & & 14 & 11.0 & a & A... & 18 & it & & 39.3 & 34 \\
\hline C. & 0 & 12 & & 12.00 & () & $k \ldots$ & $1: 1$ & & 3 & 89.5 & $\therefore .5$ \\
\hline$N$. & 7 & & 15 & $x+.01$ & $\cdots \cdots .94$ & Cat. & 24 & it & & foris & $\therefore \quad k$ \\
\hline 0 & 8 & 10 & & I 6.00 & 0 & Se... & 21 & & 73 & 44.8 & 1 \\
\hline Ii1 $\ldots .$. & 9 & & 19 & 19.0 & 0 & $-m \ldots \ldots$ & $2:$ & +4 & & & \\
\hline $\mathrm{Ne} . . .$. & IO & 20 & & 20.0 & 0 & $\ldots$ & $\therefore 3$ & & 4 & & . \\
\hline $\mathrm{Na} . .$. & II & & 2.3 & 23.05 & 0.05 & $\mathrm{Ti} \ldots$ & 24 & 48 & & 18.1 & 0 \\
\hline $\operatorname{Mg} \ldots .$. & I 2 & 24 & & $24 \cdot 36$ & 0.30 & $V \ldots$ & 25 & & 31 & $52 \ldots$ & $b$. \\
\hline Al...... & 13 & & 27 & $27 \cdot 1$ & 0.1 & Cr... & 20 & 52 & & 52.1 & 6.1 \\
\hline$\ldots$ & $I_{4}$ & 28 & & 28.4 & $11+$ & $\mathrm{Mn}$ & $2 ?$ & & 55 & $55 \cdot 0$ & $0, i$ \\
\hline & & & & & & Fe. & 28 & af & & $58 \%$ & \\
\hline
\end{tabular}

Many besides Rydberg have, of course, noticed and studied the curious deviations in the table of atomic weights and Rydberg', work is nem tioned merely because it seems unusually explicit.

The orderly arrangement of the series is striking. It will is noticed

$$
\text { : Z. anorg. Chem., 14, } 66 \text { (189i) }
$$


that in three cases only are the D's greater than unity and only in two cases are they negative.

Rydberg points out that although the heavier elements do not conform well to this scheme, i.e., do not in general give the small fractional, values of (D) noticed above, yet this is in reality no valid objection, for the numerical values of the weights of heavier elements depend much more on the value of the arbitrary unit chosen than do those of the lighter weight elements, and hence they can have little influence one way or the other in estimating the validity of the curious relations he sets forth.

The whole question is, of course, whether these differences represent real physical deviations from something or whether they are merely mathematical remainders. Rydberg certainly believes them to represent physical realities, and considering the before-mentioned overwhelming improbability that the approximation of the atomic weights to whole numbers is due to chance, we can hardly doubt that he is right.

The question will doubtless be asked why is there no loss of mass found when a violent chemical reaction takes place and energy is lost. The expression for loss of mass in terms of loss of energy, when written in the differential form (substituting $\mathrm{V}=3 \cdot 10^{10}$ ) is

$$
\Delta \mathrm{M}=\frac{4}{27} \mathrm{IO}^{-20} \Delta \mathrm{E},
$$

and if we substitute the heat of reaction of any known chemical reaction for $\Delta \mathrm{E}$, we find that $\Delta \mathrm{M}$ is too small to be detected even by the delicate experiments of Landolt.

For the reaction

$$
\mathrm{O}_{2}+2 \mathrm{H}_{2}=2 \mathrm{H}_{2} \mathrm{O}
$$

$\Delta \mathrm{M}$ for one gram molecule is about $10^{-10}$ of a gram.

In radioactive changes, however, the energy is enormously greater and hence is to be detected as before mentioned.

If we consider the whole atom is electric instead of only part, as we have considered above, a conception. which is by no means artificial since it has been proven that the mass of an electron is entirely due to its charge, we reach the interesting conclusion that on this basis the "Indestructibility of Matter" is only a corollary of the "Conservation of Energy," for if the atoms are to be considered as electrical systems it follows that the law of the Conservation of Mass, which is essentially the same as the Indestructibility of Matter, must be approximately true, though not strictly true. It must be approximately true because no known chemical or physical change, with the exception of radioactivity, involves anything but a reldtively minute liberation or absorption of energy, but the law cannot be absolutely exact, for even this minute loss of energy must involve, on the present basis, its corresponding loss of mass. Thus even 
the cooling of a hot body must involve a certain diminution of mass, though this, of course, is extraordinarily small, much smaller even than the loss due to most chemical reactions.

It would be interesting to search for other evidence of the existence of this vast store of energy in matter. Such evidence would involve effects, which would be approximately proportional to the density. Such an effect is found in the case of the absorption of the $\beta$-rays of radium when passing through different kinds of matter. Here the absorption is proportional to the density over a very wide range. Strutt has shown that in the case of fourteen substances whose absorption he measured, where the density varied from 0.007 in the case of sulphur dioxide, to $2 \mathrm{I} .5$ in the case of platinum, the ratio of the absorption to the density has an average deviation of only 20 per cent.

From a physical point of view, also, it is interesting to note that, since gravitation has always been found to be strictly proportional to mass, it follows from the above that the electrical structure of matter requires that gravitation should be proportional to the energy contained in the gravitating bodies and to this energy alone. Thus gravitation must be considered on the present basis as existing between quantities of confined, electromagnetic energy and not between "masses" in any other sense. More knowledge bearing on the electrical theory of matter would therefore throw considerable light on the outstanding mystery of gravitation.

\section{Summary.}

We have seen that, assuming the electrical theory of matter-the theory, that is, which considers the atoms as systems composed of electric charges - it follows that the mass of a piece of matter is determined solely by the amount of electromagnetic energy which it contains and is proportional to this amount. The energy in ergs which endows one gram with its mass is equal to three-fourths of the square of the velocity of light, or, in round numbers, twenty million horse-power-hours, or the energy corresponding to the work of one thousand horses working for two years. This enormous amount is by no means impossible since the amount of energy which the radioactive substances are known to lose in passing to lower forms is quite appreciable in comparison.

On the electrical theory of matter it therefore follows that the chief property of matter, the property which gives us a quantitative definition, namely its inertia or mass, is really a property of the energy stored up within the structure which defines the space relations of a piece of matter and is not a property of the structure itself. Thus it follows that the law of the "Conservation of Mass," which we have here reason to believe is only approximate, is in reality a corollary to the law of the 
"Conservation of Energy" and thus this latter law and the "Indestructibility of Matter" are closely akin.

From the above it follows that on the present basis any loss of energy must involve a decrease in mass. Thus when a chemical reaction which liberates heat has taken place, when a body is cooled or when by the process of radioactivity one substance loses energy and is transformed into another substance, there must be a decrease in the mass of the whole and hence also a decrease in weight. In the first two cases mentioned, however, the change is too small to be detected but in the last case the change should be appreciable, and we have a ready explanation of the irregularities which occur in the table of atomic weights.

Finally, it is pointed out that since gravity is proportional to mass it would appear that gravitation must be considered as acting between quantities of confined energy and not between "masses" in any other sense.

[Contribution from the Kent Chemical IAboratory, University of Chicago.]

\section{TWO NEW METHODS FOR THE DETERMINATION OF THE SECONDARY IONIZATION CONSTANTS OF DIBASIC ACIDS.}

By Herbert N. MCCOY.

Received March 3, Igo8.

The ionization of a dibasic acid, $\mathrm{H}_{2} \mathrm{X}$, takes place in two stages, represented by the following equations:

$$
\begin{aligned}
& H \cdot H X=k_{1} H_{2} X ; \\
& H \cdot X=k_{2} H X ;
\end{aligned}
$$

where $k_{1}$ and $k_{2}$ are the ionizaton constants, and where the formulae represent the molar or ionic concentrations of the corresponding substances. With the exception of a few moderately strong acids, like oxalic, all organic dibasic acids are found by conductivity measurements to dissociate essentially according to equation ( $\mathrm{I}$ ), in solutions more concentrated than milli-normal; they thus behave like monobasic acids. ${ }^{1}$ The second constant, $k_{2}$, is always much smaller than the first. While the secondary ionization produces but a negligible effect in solutions of the free acid, in solutions of the acid salts its effect is of great impor tance.

When an acid salt, like NaHX, is dissolved in water it reacts, partially, forming the free acid and the neutral salt, according to the equation, thus:

$$
{ }_{2} \mathrm{NaHX} \rightleftarrows \mathrm{H}_{2} \mathrm{X}+\mathrm{Na}_{2} \mathrm{X} \text {. }
$$

The state of equilibrium reached is governed by equations ( 1 ) and (2), which, by combination, give 